\title{
(2) OPEN ACCESS \\ Why is there variation in test ordering practices for patients presenting to the emergency department with undifferentiated chest pain? A qualitative study
}

\author{
Julie Li $\odot,{ }^{1}$ Maria R Dahm, ${ }^{1}{ }^{12}$ Judith Thomas, ${ }^{1}$ Nasir Wabe, ${ }^{1}$ Peter Smith, ${ }^{3,4}$ \\ Andrew Georgiou'
}

\begin{abstract}
Handling editor Ellen J Weber
- Additional supplemental material is published online only. To view, please visit the journal online (http://dx.doi. org/10.1136/emermed-2020211075)

${ }^{1}$ Centre for Health Systems and Safety Research, Australian Institute of Health Innovation, Macquarie University, Sydney, New South Wales, Australia ${ }^{2}$ Institute for Communication in Health Care, Australian National University College of Arts and Social Sciences, Canberra, Australian Capital Territory, Australia

${ }^{3}$ Emergency Medicine, Illawarra Shoalhaven Local Health District, Wollongong, New South Wales, Australia

${ }^{4}$ Graduate School of Medicine, University of Wollongong, Wollongong, New South Wales, Australia
\end{abstract}

\section{Correspondence to}

Julie Li, Australian Institute of Health Innovation, Macquarie University, Sydney, New South Wales 2109, Australia; julie.li@mq.edu.au

Received 20 December 2020 Accepted 13 August 2021 Published Online First 2 September 2021

Check for updates

(C) Author(s) (or their employer(s)) 2021. Re-use permitted under CC BY-NC. No commercial re-use. See rights and permissions. Published by BMJ.

To cite: Li J, Dahm MR, Thomas J, et al. Emerg Med J 2021:38:820-824.

\section{ABSTRACT}

Introduction Up to one-third of laboratory tests ordered in the ED for adults presenting with undifferentiated chest pain are generally not indicated by current Australian guidelines. This study set out to undertake a qualitative investigation of clinician perceptions to identify the reasons for variations in pathology requesting.

Methods For this study, we draw on data from semistructured interviews $(n=38)$ conducted in the EDs and laboratories across three hospitals as part of a larger study on the test result management process from test request to result follow-up. Thematic analysis was conducted to determine what aspects of the clinical routines and environment might contribute to variations in pathology requesting. Informed by the findings from the analysis, targeted questions were developed and further focus groups $(n=5)$ were held with clinicians, hospital management and electronic medical record (eMR) analysts to investigate in more detail the reasons for requesting outside of guidelines.

Results Participants cited four main reasons for ordering outside of guidelines. Clinicians requested tests outside of guidelines and the ED scope of practice to facilitate the patient journey along the broader continuum of care, including admission to hospital or transfer to another site. Clinicians were also faced with multiple and inconsistent guidelines regarding appropriate test selection. Limited access to in-house specialty and diagnostic services also influenced ordering patterns in smaller non-referral hospitals. Finally, certain features of the current electronic ordering framework within the eMR facilitated overordering and failed to impose any real restrictions on ordering inappropriately or outside of scope of practice.

Conclusion Beyond the standardisation of pathology requesting advice across electronic decision support, order sets and guidelines, attempts to address issues related to the appropriateness and variation of laboratory test ordering should consider local and systemic factors which also shape the ordering process.

\section{INTRODUCTION}

Diagnostic investigations involving laboratory tests aim to: increase certainty of the presence or absence of disease; support clinical management; and monitor a patient's trajectory (eg, during or after treatment). ${ }^{1}$ It is estimated that $60 \%-70 \%$ of medical decisions are based on laboratory test results. ${ }^{2}$ Evidence shows that some $84 \%$ of people

\section{Key messages}

What is already known on this subject

- An estimated $21 \%$ of pathology testing in healthcare is potentially redundant.

- Almost one-third of laboratory tests ordered in the ED for patients presenting with chest pain are generally not indicated by Australian guidelines.

\section{What this study adds}

- Laboratory test requesting in the ED is shaped by local and systemic factors including the requirements of admission, conflicting guidelines, availability of in-house resources and services, and characteristics of the electronic medical record ordering framework.

admitted to a hospital can expect to have at least one pathology test during their stay, usually within 4 hours of their admission as an inpatient. ${ }^{3}$

Zhi and colleagues estimated that $45 \%$ of laboratory testing in healthcare is underused and $21 \%$ overused. ${ }^{4}$ Major international initiatives, such as Choosing Wisely, are seeking to reduce the inappropriate use of tests, treatments and procedures as part of a collaborative effort involving health professionals, consumers and specialist colleges, and societies and associations. ${ }^{5}$ The Sensible Test Ordering Practice initiative was developed in Australia to promote consistent and rational diagnostic test ordering practices in acute care settings. ${ }^{6}$ It involves the use of traffic light systems (green, amber or red) to restrict the range of tests that can be ordered depending on the seniority of the clinician-'green' tests may be ordered by junior doctors, while more complex and expensive tests in 'amber' or 'red' require senior medical staff sign-off. The Australasian College for Emergency Medicine (ACEM) and the Royal College of Pathologists of Australasia (RCPA) have also developed a set of laboratory testing recommendations for adult patients attending the ED across a range of common presentations.

A retrospective evaluation of variation in requesting potentially avoidable tests for adult patients presenting to six EDs with undifferentiated chest pain found that almost one-third of tests ordered in the ED for these patients were 'generally not indicated' (GNI) by the joint ACEM/ 


\begin{tabular}{|c|c|}
\hline Recommendation & Test \\
\hline Perform test & $\begin{array}{l}\text { Electrolytes/urea/creatinine, glucose, troponin, full } \\
\text { blood count }\end{array}$ \\
\hline Consider or ask supervisor & D-dimer, liver function test \\
\hline Generally not indicated & $\begin{array}{l}\text { All tests not listed in the categories above, eg, blood } \\
\text { cultures, coagulation studies, calcium phosphate } \\
\text { albumin, urate, lipase, C reactive protein, creatine } \\
\text { kinase, drug level, blood group/antibody screen, } \\
\text { blood gas, urinalysis }\end{array}$ \\
\hline
\end{tabular}

ACEM, Australasian College for Emergency Medicine; RCPA, Royal College of

Pathologists of Australasia.

RCPA guidelines. ${ }^{8}$ A summary of the ACEM/RCPA pathology requesting guidelines for undifferentiated chest pain is presented in table $1 .^{7}$ In light of such findings, the current study set out to undertake a qualitative investigation to identify possible reasons for GNI tests.

\section{METHODS}

\section{Interviews}

As part of a larger study investigating safe and effective test result management, we conducted ethnographic observations and semistructured interviews with ED patients and clinicians, intensive care unit clinicians, and staff in the laboratory and medical imaging departments across three hospital sites between October 2016 and November 2017. Qualitative research methods are effective for in-depth explorations of clinician perceptions regarding clinical work practices and experiences in real-life settings. ${ }^{9}$ Interview questions were informed by the literature and included all aspects of the test management cycle including test requesting practices. A purposive sample of participants involved in test result management was selected in consultation with departmental management at each site to include a cross-section of roles and experience. Recruitment concluded when interviews ceased yielding new information related to the test result management process.

For this study, we drew on data from semistructured interviews $(n=38)$ conducted with clinicians in the EDs and laboratory staff given their involvement in the test requesting process. Detailed data collection and analysis of the larger dataset are reported elsewhere. ${ }^{10}$ Patients were not involved in this study.

Thematic analysis ${ }^{11}$ drawing from a grounded theory approach $^{12}$ was conducted on coded data (NVivo V.11; QSR International) from the larger study to determine what aspects of clinical work and environment might contribute to GNI test requesting practices. Two experienced qualitative researchers who had collected the data but had no previous engagement with the sites (MRD, JL) extracted all data coded at the node 'ordering process' and ran a broad context keyword query in NVivo to capture all references to 'chest pain', or any of the top five identified GNI tests (coagulation studies, calcium magnesium phosphate, blood gas, lipase and $\mathrm{C}$ reactive protein). ${ }^{8}$ The same researchers individually analysed the data to develop themes using an inductive, iterative process of initial open coding, constant coding comparison and development of categories from open codes. Individually identified themes were presented and refined in discussion between the research team.

\section{Focus groups}

Informed by the findings from the thematic analysis, we developed targeted questions to investigate the reasons underlying the ordering of GNI tests (online supplemental file 1). Key informants most likely to be knowledgeable about the topic were identified by researchers and clinicians who participated in the initial interviews. We then conducted further focus groups via teleconference with these nominated experts in May 2019. These comprised clinical and managerial hospital executives from the same EDs and electronic medical record (eMR) analysts responsible for overseeing the eMR system for the district which included all three hospitals. Focus group members were emailed a summary of findings with select quotes prior to the focus groups to guide discussion on the day. Focus groups were digitally audio-recorded, transcribed verbatim, de-identified and assigned site and participant codes in preparation for analysis in NVivo. Transcripts were analysed following the principles of thematic analysis outlined above.

Trustworthiness of results was ensured through triangulation between two researchers involved in data collection across both phases of the study (MRD, JL), feedback of findings from the first phase of data analysis (interviews) to site representatives in the focus groups and review of final results by a focus group participant. Demographics of the study sites and participants are presented in table 2 .

\section{RESULTS}

Overall, staff were aware of the need for rationalised test ordering and reported that they predominantly requested tests that they felt were relevant to each patient's ED presentation. However, adherence to sensible test ordering recommendations was affected by a number of local and systemic factors including the need to facilitate the patient journey beyond the ED, multiple and inconsistent guidelines, limited access to in-house specialty and diagnostic services, and various characteristics of the electronic ordering framework within the eMR.

\section{Facilitating patient admission}

Clinicians overwhelmingly reported requesting pathology related to patient care within the $\mathrm{ED}$, including investigations whose results returned before a patient leaves, but also tests with longer turnaround times such as microbiology. However, ED clinicians also placed requests deemed beyond a patient's ED encounter for a number of reasons. Clinicians would frequently request tests under instruction from interdepartmental specialists to streamline workflow.

It's become a very common culture in emergency, that the external teams, when you ring to let them know about a patient, they will say, "Oh, can you just order this, this, this and this, I'm a bit delayed for a couple of hours. So, if you order that for me, I'll come down and see them when I can." There is a lot of that. (Nurse practitioner, site 1 (focus group))

Clinicians in the ED also reported requesting laboratory tests in anticipation of tests required by the admitting team to facilitate admission or to reduce lead-up times for investigations with longer wait times.

Certainly some tests you'll order that won't necessarily help us in the emergency department, but are for the inpatient team, so if you know a patient is going to be a surgical patient, you might do a CRP [C reactive protein] which doesn't really mean anything to us whatsoever and we don't really want it, but you know that the surgeons are going to ask for it, so you do it just to facilitate referrals. (Consultant, site 2 (interviews)) 


\begin{tabular}{|c|c|}
\hline (Site \#) hospital site & $\begin{array}{l}\text { Annual ED } \\
\text { presentations* }\end{array}$ \\
\hline (1) major metropolitan & 64193 \\
\hline (2) large regional & 38796 \\
\hline (3) medium metropolitan & 29832 \\
\hline Participants-interviews & Total \\
\hline $\begin{array}{l}\text { Senior medical } \\
\text { (medical directors, senior emergency medicine physicians, career } \\
\text { medical officerst) }\end{array}$ & $\mathrm{n}=11$ \\
\hline $\begin{array}{l}\text { Junior medical } \\
\text { (interns, residents, registrars) }\end{array}$ & $\mathrm{n}=7$ \\
\hline $\begin{array}{l}\text { Nursing } \\
\text { (registered nurses, advanced practice nurses, nurse practitioners) }\end{array}$ & $\mathrm{n}=8$ \\
\hline $\begin{array}{l}\text { Laboratory } \\
\text { (staff specialists, managers, scientists, typists, technical officers) }\end{array}$ & $\mathrm{n}=12$ \\
\hline Participants-focus groups & Total \\
\hline Clinical (medical and service directors, nurse practitioner) & $n=3$ \\
\hline eMR (senior analysts) & $\mathrm{n}=2$ \\
\hline
\end{tabular}

*Calculated using ED presentation data Oct 2016-Sept 2017 from the Health Information Exchange, NSW Health.

†A physician who has completed their training without any area of specialisation and may work in a variety of clinical settings in a hospital. ${ }^{23}$

eMR, electronic medical record; NSW, New South Wales.

Overall, large numbers of GNI tests in the ED were the corollary of ordering outside the ED scope of practice to facilitate the entire patient journey.

We knew that when we started frontloading [tests] that we would be over-ordering. And that's the cost that you have to bear given the need for maintenance of flow and efficiency. (Consultant, site 3 (focus group))

So, it's often like a team sharing, I guess you could think of it. We bear the cost and we do the work but it's not necessarily for their emergent point of care, it's for their ongoing care of their community care or to link in with all those other services. [...] If they've got an appointment tomorrow, we'll still discharge them [...] but we've done the whole work up for them. (Nurse practitioner, site 1 (focus group))

\section{Conflicting guidelines}

In addition to national (ACEM/RCPA) and local district-level guidelines for test ordering such as ordering protocols for advanced care nurses, test requesting advice was also embedded in decision support in the eMR. Participants reported three types of ordering aids: (1) order sets for particular presenting problems or symptoms (eg, chest pain); (2) electronic clinical patient management pathway tools and (3) the colour-coded 'Quick Orders' (traffic light) screen. Participants commented on issues associated with consistency across the different guidelines.

If you then compare [the order set for ischaemic chest pain] with the clinical pathway that's on eMR, there are additions to that which aren't necessarily part of the order set so you've got inconsistency there. (Consultant, site 3 (focus group))

Tests contained within order sets and nursing protocols also included tests that were GNI in the best practice guidelines. For example, coagulation studies and a blood group and antibody screen were indicated in the advanced nursing protocol and chest pain pathway for patients receiving or likely to require anticoagulation therapy while these tests are GNI in the ACEM/ RCPA guidelines (which propose consideration of a D-dimer only). Lipase, another GNI test, was reported by nursing staff as a 'green test' on the eMR traffic light order screen. Glucose, a recommended test for chest pain presentations, was also said to be automatically clustered by default with calcium magnesium phosphate (a GNI test) in the eMR.

Awareness of the tests which made up the different order sets also varied, with some clinical staff admitting being unsure of what was included, and of the national best practice guidelines themselves.

I mean I know the [ACEM/RCPA] guidelines are there but, do I know what they are specifically? Have I read them at some stage in the past? Probably. But no. (Nurse practitioner, site 1 (focus group))

Additionally, junior and senior doctors differed in their perception of the usefulness of order sets in the ED. Senior clinicians mentioned that order sets typically cast a very broad net and thus were known to result in the potential overordering of tests. Conversely, junior doctors valued order sets for the opportunity to rule a diagnosis in or out.

I just use the order sets [...] I'm pretty happy with all of them because $[. .$.$] you don't really know what's going on, so, you want to$ look at everything rather than miss something, especially in emergency. It's just the nature of our work, we have to cover all the bases. (Registrar, site 1 (interviews))

It's not unusual for clinicians within a specific unit to use those order sets $[\ldots]$ But from the emergency department perspective, presentation is a symptom. [...] There's no order set that's going to be appropriate for that. It has to go down the traditional path of you do a history, you do an examination, you have some thought process about what might be going on, and you evaluate that with your pre-test probability, and then use the test to give you a greater understanding of your post-test probability. But using the test as your only evaluation is where we go wrong with this, and that's why the order sets are, I think, challenging, and inappropriately used. (Consultant, site 3 (interviews))

\section{Limited in-house specialty and diagnostic services}

A senior ED physician from a smaller metropolitan hospital indicated that limited after-hours access to diagnostic services resulted in workarounds, and increased ordering of certain GNI tests.

Clearly the [blood] gas is flat-out stand-up [...] A gas in my institution is a default biochemistry because after hours, everything goes to [another site] and there's a long turnaround time [...] It gets to that stage now that anyone that gets cannulated almost invariably gets a gas and that's borne out of this practice of knowing that after hours the gas is the only thing you've got rapid accessibility to. (Consultant, site 3 (focus group))

The same physician also suggested that smaller sites were required to complete a more thorough diagnostic workup for patients to ensure acceptance by referral hospitals.

This department is challenged by the lack of inpatient services that the hospital provides. [...] The specialty patients, or the subspecialty patients [...] all that goes to [another hospital]. Before [that hospital] tend to accept, they need to be fairly certain that what it is they're accepting is appropriate for them. So there's often a greater workup here for the patients we need to transfer, to ensure that they get specificity at their end of the world. (Consultant, site 3 (interviews))

\section{Impact of health information technology on inappropriate ordering}

Several participants felt that the introduction of eMR alone had made test requesting easier, resulting in increased test ordering; 
the ease of selecting a set of tests in an order set, or having a list of commonly ordered tests on a 'common orders' screen created greater potential for increased pathology requests compared with the previous practice of listing individual tests manually in a paper system.

Handwritten [requests] are less, because they have to think about what they actually want rather than just having a list in front of them $[\ldots]$ and not really necessarily thinking about the clinical situation and what it is $[\ldots]$ they just tick the box. (Senior laboratory scientist, site 1 (interviews))

ED clinicians commented that the multiple order screens within the eMR made it difficult to establish a common ordering process, while the 'Quick Order' (traffic light) screen was not intuitive, ever changing and time-consuming to navigate, deterring the use of such features designed to facilitate sensible test ordering.

\begin{abstract}
They're not set up as ordering sets. They're set up to try and get people to think about whether they should order the test. Which is completely appropriate, and I agree with the concept, I don't quite agree with the way it's rolled out. [...] what it's done is made it harder and slower to find the tests. It's quicker to do it in the front screen than it is to do it in the traffic light screen. (Consultant, site 2 (interviews))
\end{abstract}

Further, the eMR did not impose any systematic barriers on pathology requested by junior doctors or nursing staff. For example, nurses were theoretically confined to order within their scope of practice, or in adherence to nursing protocols which were specific, institutionally approved written procedures prescribing nursing actions in given situations. However, they often ordered out-of-scope tests for senior staff under verbal orders which did not require immediate electronic sign-off by a physician for the order to proceed. Repeated verbal orders for certain tests, such as $\mathrm{C}$ reactive protein, created a culture where nurses ordered some tests by default without receiving instruction from physicians.

[Nurses] have restrictions on what they can order off their own bat but by asking for a verbal order, they can order anything [...] And quite clearly not talking to us. The problem is there are lots of systems in place for various things, but there are easy workarounds to all of them. (Consultant, site 2 (interviews))

What is frustrating and [...] it may contribute to this added number of tests that seem to be outside the guidelines is that the nursing staff in their ACN [Advanced Clinical Nurse] role are often requesting tests that are outside of their guidelines. [...] the justification is that it's something that the medical staff are requesting anyway so [they'll] do it in advance, and it becomes this sort of self-perpetuating result. (Consultant, site 3 (focus group))

\section{DISCUSSION}

Findings from this study indicate that while the act of pathology requesting occurs at the individual level, test requesting patterns in the ED are also shaped by local and systemic factors, including: requirements of admission, existing guidelines, availability of in-house resources and services, and characteristics of the current electronic ordering framework in the eMR. The diagnostic process is a series of tasks involving multiple providers across and between institutions. ${ }^{13}$ Described as the 'front door' or 'gatekeeper' of the hospital, care of the ED patient extends beyond diagnosis and treatment within the department to the coordination and negotiation of the patient trajectory through the hospital or beyond. ${ }^{14}$ As such, it is not uncommon for pathology requesting decisions to include tests that are out of scope for ED practice but are relevant for other phases of patient care. $^{15}$

Existing literature has also reported that smaller and/or more regional EDs were more likely to order inappropriate tests than larger and/or metropolitan EDs. ${ }^{8}$ Participants in our study perceived that non-referral and regional hospitals were required to complete more thorough pathology investigations before referral hospitals were satisfied that a patient transfer was truly warranted.

Numerous interventions have been introduced to reduce inappropriate laboratory testing, with varying degrees of success. Interventions based on eMRs offer pertinent information for clinical decision-making, such as guideline-based alerts and decision support. ${ }^{16}$ While order sets may lighten the cognitive load, they can also facilitate overordering. ${ }^{17}$ As reflected in previous studies, junior medical staff viewed the utility of routine order sets in the eMR more favourably than their senior counterparts. ${ }^{18}$ However, despite criticisms of the relevance and use of order sets from senior physicians, there were discrepancies between the ACEM/RCPA guidelines with both district-level recommendations and eMR-based decision support, with some participants struggling to recall and reconcile inconsistencies between the different guidelines. Without harmonisation, these inconsistencies potentially negate the intended purpose of such recommendations in effective test selection and interpretation. ${ }^{19} 20$

The availability of data and information in the eMR is crucial to diagnosis, but how they are presented and the information technology (IT) demands of the user may determine whether they help or hinder the process. ${ }^{21}$ Difficulties navigating the 'Quick Orders' traffic light screen deterred the use of such an ordering aid. Protocols around the nursing scope of practice formally restricted nurses in what they could order independently, however the current electronic ordering framework imposed no real barrier against ordering outside their scope of practice. If health IT is to provide optimum clinician support, it must integrate with organisational workflow in a way that aids the cognitive work of users and the specific needs of the context in which it will be used. ${ }^{22}$

The study has limitations in that we did not interview all clinicians who engaged in the laboratory test requesting process in the EDs, instead exploring the topic further with an 'expert panel' of participants. Requesting behaviours might be motivated by different influences across provider categories and the limited representation by each category in our sample might have been insufficient to capture all such influences. Focus groups comprised senior clinicians, managerial and eMR staff who are appropriate for providing rich insight into the contextual factors most likely to affect clinical work processes. However, factors affecting test requesting behaviour will further vary between sites and regions, limiting generalisability of findings nationally and globally.

Evidence from this study suggests that attempts to address issues related to the appropriateness and variation of laboratory test ordering could benefit from the establishment of consistent, standardised laboratory requesting advice across electronic decision support, order sets and guidelines to facilitate best practice in laboratory test requesting. Beyond decision support and requesting advice, key local and systemic factors also shape the ordering process. The role of the ED near the commencement of a patient's healthcare journey fosters test requesting practices that aim to expedite and streamline the patient care journey along the continuum of care. Default requesting patterns of remote or smaller, non-referral hospitals are, in part, influenced by the requirements of referral hospitals and availability 
of in-house diagnostic services. Finally, the usability of health IT interventions and their ability to positively affect provider requesting behaviour require a robust level of integration with clinical workflows and the specific necessities of the context in which they will be used.

Twitter Julie Li @DxInformatics

Contributors AG and MRD were involved in the conceptualisation and design of the study. JL and MRD performed data collection, and JL, MRD, JT, NW, PS and AG were involved in the analysis and/or interpretation of the data. JL drafted the article, and MRD, JT, NW, PS and AG critically reviewed the article for important intellectual content. All authors approved the article.

Funding This study was funded by the National Health and Medical Research Council (Partnership Project Grant number 1111925).

Competing interests None declared.

Patient consent for publication Not required.

Ethics approval Ethical approval was granted by the South Eastern Sydney Local Health District Human Research Ethics Committee (HREC/16/POWH/412).

Provenance and peer review Not commissioned; externally peer reviewed.

Data availability statement No data are available. Data collection from the relevant Local Health Districts during this study was granted under ethical approval. Local Health District data are not publicly available. Data collection from the relevant Local Health Districts during this study was granted under ethical approval. Local Health District data are not publicly available.

Supplemental material This content has been supplied by the author(s). It has not been vetted by BMJ Publishing Group Limited (BMJ) and may not have been peer-reviewed. Any opinions or recommendations discussed are solely those of the author(s) and are not endorsed by BMJ. BMJ disclaims all liability and responsibility arising from any reliance placed on the content. Where the content includes any translated material, BMJ does not warrant the accuracy and reliability of the translations (including but not limited to local regulations, clinical guidelines, terminology, drug names and drug dosages), and is not responsible for any error and/or omissions arising from translation and adaptation or otherwise.

Open access This is an open access article distributed in accordance with the Creative Commons Attribution Non Commercial (CC BY-NC 4.0) license, which permits others to distribute, remix, adapt, build upon this work non-commercially, and license their derivative works on different terms, provided the original work is properly cited, appropriate credit is given, any changes made indicated, and the use is non-commercial. See: http://creativecommons.org/licenses/by-nc/4.0/.

ORCID iD

Julie Li http://orcid.org/0000-0002-8881-5962

\section{REFERENCES}

1 Knottnerus JA, van Weel C, Muris JW. Evidence base of clinical diagnosis: evaluation of diagnostic procedures. BMJ 2002;324:477-80.

2 Forsman RW. Why is the laboratory an afterthought for managed care organizations? Clin Chem 1996:42:813-6.
3 Georgiou A, Sezgin G, Li L, et al. Who gets a laboratory test in hospital, why, and how often? A retrospective observational study of 4 Australian hospitals. J Appl Lab Med 2019:4:343-54.

4 Zhi M, Ding EL, Theisen-Toupal J, et al. The landscape of inappropriate laboratory testing: a 15-year meta-analysis. PLoS One 2013;8:e78962.

5 Levinson W, Kallewaard M, Bhatia RS, et al. 'Choosing wisely': a growing international campaign. BMJ Qual Saf 2015;24:167-74.

6 Emergency Care Institute New South Wales. Ed quality framework sensible test ordering, 2018. Available: https://aci.health.nsw.gov.au/networks/eci/administration/ ed-qf-project/ed-qf-sensible-test-ordering [Accessed 04 Jun 2020].

7 Australasian College for Emergency Medicine and the Royal College of Pathologists of Australasia. Guideline on pathology testing in the emergency department, 2018. Available: https://acem.org.au/getmedia/57501811-e932-4c74-85be-159f0621917f/ RCPA-ACEM-Guideline-v01-(Mar-13)-Final.aspx [Accessed 06 Jun 2020].

8 Wabe N, Dahm MR, Li L, et al. An evaluation of variation in pathology investigations and associated factors for adult patients presenting to emergency departments with chest pain: an observational study. Int I Clin Pract 2018:e13305.

9 Pope C, Mays N. Qualitative methods in health research. Qualitative research in health care 2006:3:1-11.

10 Thomas J, Dahm MR, Li J, et al. Variation in electronic test results management and its implications for patient safety: a multisite investigation. J Am Med Inform Assoc 2020:27:1214-24

11 Crabtree BF, Miller WL. Doing qualitative research. Sage Publications, 1999.

12 Corbin J, Strauss A. Basics of qualitative research: techniques and procedures for developing grounded theory. Sage publications, 2014.

13 National Academies of Sciences Engineering and Medicine. Improving diagnosis in health care. Washington DC: National Academies Press, 2015.

14 Nugus $\mathrm{P}$, Braithwaite J. The dynamic interaction of quality and efficiency in the emergency department: Squaring the circle? Soc Sci Med 2010;70:511-7.

15 Rehmani R, Amanullah S. Analysis of blood tests in the emergency department of a tertiary care hospital. Postgrad Med J 1999;75:662-6.

16 Georgiou A, Li J, Thomas J, et al. The impact of health information technology on the management and follow-up of test results - a systematic review. J Am Med Inform Assoc 2019;26:678-88

17 Leis B, Frost A, Bryce R, et al. Standard admission order sets promote ordering of unnecessary investigations: a quasi-randomised evaluation in a simulated setting. BMJ Qual Saf 2017;26:938-40.

18 Cadamuro J, Gaksch M, Wiedemann H, et al. Are laboratory tests always needed? Frequency and causes of laboratory overuse in a hospital setting. Clin Biochem 2018;54:85-91.

19 Hickner J, Thompson PJ, Wilkinson T, et al. Primary care physicians' challenges in ordering clinical laboratory tests and interpreting results. J Am Board Fam Med 2014;27:268-74.

20 Committee on Patient Safety and Health Information Technology; Institute of Medicine. Health it and patient safety: building safer systems for better care. Washington DC, USA: National Academies Press, 2011: 220.

21 Carr S. Health it and diagnostic safety: promise and peril. Improve Diagnosis 2015:2:1-4.

22 Carayon P, Karsh BT, Cartmill R. Incorporating health it into workflow redesign: Request for information summary report. Rockville MD, USA: Agency for Healthcare Research and Quality, 2010.

23 NSW Government. Training and working as a doctor in the NSW public health system. Available: https://www.mapmycareer.health.nsw.gov.au/Pages/training-and-workingas-a-doctor-in-the-NSW-public-health-system. aspx?section=ms [Accessed $21 \mathrm{Apr}$ 2021]. 